\title{
FUNCTIONS OF COPRIME DIVISORS OF INTEGERS
}

\author{
BY E. T. BELL
}

1. Unique Decompositions. If a set $U$ of distinct positive integers $1, u_{1}, u_{2}, \cdots$ is such that*

$$
\left(u_{i}, u_{j}\right)=1, \quad i \neq j, \quad i, j=1,2, \cdots,
$$

we call $U$ a coprime set. If to $U$ we adjoin all positive integral powers $u_{1}^{\alpha_{1}}, u_{2}{ }^{\alpha_{2}}, \cdots, \alpha_{1}>0, \alpha_{2}>0, \cdots$ of integers in $U$, we get the extended set $E(U)$. If $m$ is in $E(U)$, we call $m$ a $U$-integer.

THEOREM 1. If $n>1$ is representable as a product of powers of integers $>1$ in $U$, the representation is unique (up to permutations of the factors), say

$$
n=u_{1}^{c_{1}} \cdots u_{r}^{c_{r}}, \quad u_{i}>1, \quad c_{i}>0, \quad i=1, \cdots, r .
$$

For, by the definition of $U$, the $u_{i}$ in (2) are distinct, and by (1) a prime $p$ such that $p \mid n$ is such that $p \mid u_{j}$ for precisely one $j$, $0<j \leqq r$. We call (2) the $U$-decomposition of $n$.

Obviously there exist $U$ 's such that some $n>1$ are not $U$-decomposable. From the fundamental theorem of arithmetic we have the following theorem:

Theorem 2. If $P \equiv p_{1}, p_{2}, \cdots$ is the set of all positive primes, the only $U$ such that every integer $n>1$ is $U$-decomposable is $U \equiv P$.

We shall consider also another type of unique decomposition, valid for all $n>1$, which has the distinguishing property of $U$-decomposition as in (2), namely, every $n>1$ is uniquely a product of powers of coprime integers $>1$.

If the integer $s>0$ is divisible by the square of no prime, we call $s$ simple. Let $S \equiv 1, s_{1}, s_{2}, \cdots$ be the set of all distinct simple integers; $S$ includes $P$ and is not a coprime set. Without confusion we may denote by $E(S)$ the set obtained by adjoining to $S$ all positive integral powers $s_{1}{ }^{\alpha_{1}}, s_{2}{ }^{\alpha_{2}}, \cdots, \alpha_{1}>0, \alpha_{2}>0, \cdots$, of simple integers.

Let $n=p_{1}{ }^{a_{1}} \cdots, p_{r}{ }^{a_{r}}$ be the $P$-decomposition of $n$. If $a_{1}, \cdots, a_{r}$ are all different, this is by definition also the $S$-decomposition. If

* In the customary notations, $(m, n)$ is the G.C.D. of $m, n$, and $m \mid n$ signifies that $m$ divides $n$ arithmetically. 
$a_{1}, \cdots, a_{r}$ are not all different, let $\alpha_{1}, \cdots, \alpha_{j}$ be all the unequal integers among $a_{1}, \cdots, a_{r}$, and let $a_{i 1}, \cdots, a_{i q_{i}}$ be all those of the $a_{1}, \cdots, a_{r}$ equal to $\alpha_{i}$. Writing $s_{i} \equiv p_{i 1} \cdots p_{i q_{i}}$, we have $n=s_{1}{ }^{\alpha_{1}} \cdots s_{j}{ }^{\alpha_{j}}$, and this, the $S$-decomposition of $n$, is unique.

THEOREM 3. Every integer $n>1$ is uniquely a product of positive integer powers of coprime simple integers $>1$; the unicity is attained when the exponents of the powers are required to be all different.

Note that since $E(S)$ contains $E(P)$, every $n>1$ has two decompositions, which coincide only if the exponents are all different, into a product of powers of coprime simple numbers, the $P$-decomposition and the $S$-decomposition as above defined, both of which are unique. Thus if $n=2^{2} \cdot 3^{2} \cdot 5^{3} \cdot 7 \cdot 11^{3}$, this is the $P$-decomposition, while $n=(2 \cdot 3)^{2} \cdot 7 \cdot(5 \cdot 11)^{3}$ is the $S$ decomposition, from the coprime simple integers $2 \cdot 3,7,5 \cdot 11$, with the respective exponents $2,1,3$, all different.

2. U-divisors, S-divisors. Referring to (2) we define the $\left(c_{1}+1\right) \cdots\left(c_{r}+1\right)$ integers

$$
u_{1}^{k_{1}} \cdots u_{r}^{k_{r}}, \quad 0 \leqq k_{i} \leqq c_{i}, \quad i=1, \cdots, r,
$$

to be the $U$-divisors of the $n$ in (2). If $m$ is a $U$-divisor of $n$ we write $(m \mid n)_{U}$. Similarly, if

$$
s=s_{1}^{a_{1}} \ldots s_{t}^{a_{t}}
$$

is the $S$-decomposition of $s$, the $S$-divisors of $s$ are the $\left(a_{1}+1\right)$ ... $\left(a_{t}+1\right)$ in tegers

$$
s_{1}^{j_{1}} \cdots s_{t}^{j_{t}}, \quad 0 \leqq j_{i} \leqq a_{i}, \quad i=1, \cdots, t .
$$

By an obvious change of notation everything defined next for $U$ is defined also for $S$, and we need state only the definitions for $U$.

If $(m \mid n)_{U}$, there is a unique $U$-divisor $t$ of $n$ such that $m t=n$; $m, t$ are conjugate $U$-divisors of $n$.

If $(d \mid m)_{U},(d \mid n)_{U}, d$ is a common $U$-divisor of $m, n$. If $g$ is a common $U$-divisor of $m, n$ which is such that $(d \mid g)_{U}$ for every common $U$-divisor $d$ of $m, n$, then $g$ is unique, and we call $g \equiv(m, n)_{U}$ the greatest common $U$-divisor of $m, n$. 
If $m, n, t$ are $U$-integers such that $m=n t$, and hence $(n \mid m)_{U}$, $(t \mid m)_{U}$, we call $m$ a $U$-multiple of $n$ (or of $t$ ). The $U$-integers $m, n$ determine a unique $U$-integer $l \equiv\{m, n\}_{U}$, the least common $U$-multiple of $m, n$, such that if $(m \mid e)_{U}$ and $(n \mid e)_{U}$, then $(l \mid e)_{U}$.

Theorem 4. If $m, n$ are $U$-integers,

$$
(m, n)_{U}\{m, n\}_{U}=m n .
$$

This is proved as in $E(P)$. Let $u_{a}, \cdots, u_{d}$ be all the integers $u_{\alpha}, \cdots, u_{\beta}, u_{\gamma}, \cdots, u_{\delta}$ in $U$ occurring in the $U$-decompositions as in (2) of the $U$-integers $m, n, m n \neq 1$. Then we may write

$$
\begin{aligned}
m & =u_{\alpha}^{\gamma_{\alpha}} \cdots u_{\beta}^{\gamma_{\beta}}=u_{a}^{h_{a}} \cdots u_{d}^{h_{d}}, \\
n & =u_{\gamma}^{t_{\gamma}} \cdots u_{\delta}^{t_{\delta}}=u_{a}^{k_{a}} \cdots u_{d}^{k_{d}},
\end{aligned}
$$

in which some of the $h, k$ may be zero. Writing $\max \left(h_{i}, k_{i}\right)=g_{i}$, $\min \left(h_{i}, k_{i}\right)=l_{i}$, we have

$$
(m, n)_{U}=u_{a}^{l_{a}} \cdots u_{d}^{l_{d}}, \quad\{m, n\}_{U}=u_{a}^{g_{a}} \cdots u_{d}^{g_{d}},
$$

and hence the theorem.

If $(m, n)_{U}=1$, then $m, n$ are called $U$-coprime.

In what follows, the particular $U$-divisors $u_{1}, \cdots, u_{r}$ of $n$ as in (2) play the part of the distinct primes dividing $n$ in the $P$-decomposition; $u_{1}, \cdots, u_{r}$ will be called the primitive $U$-divisors of $n$. By a previous remark, primitive $S$-divisors are therefore also defined.

3. Functions of $U$-divisors. By a change of notation, everything stated for $U$ holds for $P, S$, as in $\S 2$. The numerous functions depending on the $P$-decomposition of integers that occur in the theory of numbers, ${ }^{*}$ together with all of their properties depending only on the fact that the $P$-decomposition is unique (into a product of powers of coprime integers), go over unchanged to the like for $U$-decompositions by a few obvious changes in notation and terminology. We take first the example that started the entire theory of such functions for $P$.

Let $n=p_{1} a_{1} \ldots p_{r}{ }^{a_{r}}$ be the $P$-decomposition of $n$. Then the number $\phi(n)$ of integers $<n$ and prime to $n$ is

(6) $\phi(n)=n\left(1-\frac{1}{p_{1}}\right) \cdots\left(1-\frac{1}{p_{r}}\right), \quad \phi(1) \equiv 1$.

* See Dickson's History of the Theory of Numbers, vol. 1, 1919, chapters 5, 10, 19; also the writer's Algebraic Arithmetic, 1927. 
The corresponding theorem for $U$ is as follows. Let $m=u_{1}{ }^{c_{1}} \ldots$ $u_{t}{ }^{c}{ }^{t}$ be the $U$-decomposition of $m$. Then the number $\phi_{U}(m)$ of integers $<m$ and not divisible by any one of the primitive $U$ divisors $u_{1}, \cdots, u_{t}$ of $m$ is

$$
\phi_{U}(m)=m\left(1-\frac{1}{u_{1}}\right) \cdots\left(1-\frac{1}{u_{t}}\right), \quad \phi_{U}(1) \equiv 1 .
$$

The proof of (7) is precisely similar to that of (6) by means of the principle of cross-classification, ${ }^{*}$ with the remark that $(a, b)=1, a|k, b| k$ together imply $a b \mid k$. From the explicit form of $\phi(n)$ in (6), a common algebraic proof gives Gauss' result

$$
\sum \phi(d)=n, \quad d \mid n .
$$

Hence (7) implies

$$
\sum \phi_{U}(t)=m, \quad(t \mid m)_{U} .
$$

Generally, to pass from $P$ to $U, P \rightarrow U$, we have

$$
\begin{aligned}
P & \rightarrow U, \\
\text { "prime" } & \rightarrow \text { "primitive", } \\
\text { "divisor" } & \rightarrow \text { "U-divisor", } \\
(m, n) & \rightarrow(m, n)_{U} .
\end{aligned}
$$

If $f(x)$ is single-valued and finite for integer values $>0$ of $x$, $f(x)$ is (as usual) called a numerical function of $x$. The unit numerical function $\eta(x)$ is defined by $\eta(1)=1, \eta(x)=0, x \neq 1$. A generalization of Dedekind's inversion formula, proved in a previous paper, $\dagger$ is of great use in the algebra of numerical functions. If, and only if, $f(1) \neq 0$ there exists a unique numerical function $f^{\prime}(x)$, such that

$$
\sum f(d) f^{\prime}(\delta)=\eta(n), \quad n=1,2, \cdots,
$$

the sum referring to all pairs $d, \delta$ of conjugate $P$-divisors of $n$. Passing from $P$ to $U$ by means of (10), we get the theorem corresponding to (11) on making the single change $U$ for $P$ in the

* First stated as a general principle in arithmetic, apparently, by da Silva in 1854, Memorias da Academia Real das Sciencias de Lisboa, N.S.I., pp. 8-9; see Dickson, loc. cit., p. 119. A special form of the principle was noted in 1857 by H. J. S. Smith; see his Collected Mathematical Papers, vol. 1, p. 36.

$\dagger$ Tôhoku Mathematical Journal, vol. 17 (1920), pp. 221-231. Simplified proof, ibid., vol. 43 (1937), pp. 77-78. 
foregoing statement. The proof may be given precisely as in the references cited. The generalization mentioned is as follows. If $f(x), g(x), h(x)$ are numerical functions such that

$$
\begin{aligned}
& \sum f(d) g(\delta)=h(n), \quad n=1,2, \cdots, \quad g(1) \neq 0, \\
& \sum g(d) g^{\prime}(\delta)=\eta(n), \quad n=1,2, \cdots,
\end{aligned}
$$

then

$$
f(n)=\sum g^{\prime}(d) h(\delta), \quad n=1,2, \cdots,
$$

all sums referring to all pairs $d, \delta$ of conjugate $P$-divisors of $n$. If $g(n) \equiv u(n),=1$ for $n=1,2, \cdots, g^{\prime}$ is Möbius' $\mu$, and (12) becomes Dedekind's inversion. To pass from (12) to its $U$-correspondent it suffices to replace $P$ by $U$ as for (11). The $U$-correspondent of Dedekind's inversion is obtained by replacing "conjugate $P$-divisors" by "conjugate $U$-divisors," and $\mu$ by $\mu_{U}$, where $\mu_{U}(n)$ is zero if $n$ is divisible by the square of any primitive $U$-integer, and otherwise is +1 or -1 according as $n$ is the product of an even or an odd number of primitive $U$-divisors of $n$; by convention $\mu_{U}(1)=1$. A similar convention holds for any $f_{U}(n)$ which is not otherwise defined when $n=1$, namely, $f_{U}(1)=1$.

In previous papers* the algebra of numerical functions based on $P$-decomposition was constructed from (11), (12). It follows that there is a simply isomorphic algebra for any $U$-decomposition. In the $P$-algebra it was noted that the theorems hold for any set in which there is a unique decomposition. Hence the like is true for $U$-decompositions in such a set, for example the ideals of an algebraic number field.

California Institute of Technology

* Some are listed in my paper, Journal of the Indian Mathematical Society, vol. 17 (1927-28), pp. 249-260. 